\title{
Optimisation and validation of a sensitive bioanalytical method for
}

\section{niclosamide.}

Usman Arshad ${ }^{1,3}$, Henry Pertinez ${ }^{1,3}$, Helen Box ${ }^{1,3}$, Lee Tatham ${ }^{1,3}$, Rajith KR Rajoli ${ }^{1,3}$, Megan Neary ${ }^{1,3}$, Joanne Sharp $^{1,3}$, Anthony Valentijn ${ }^{1,3}$, James Hobson ${ }^{2,3}$, Catherine Unsworth ${ }^{2,3}$, Andrew Dwyer ${ }^{2,3}$, Alison Savage ${ }^{2,3}$, Tom O Mcdonald ${ }^{2,3}$, Steve P Rannard ${ }^{2,3}$, Paul Curley ${ }^{1,3}$, Andrew Owen ${ }^{1,3}$.

${ }^{1}$ Department of Pharmacology and Therapeutics, University of Liverpool, Liverpool, L7 3NY, UK

${ }^{2}$ Department of Chemistry, University of Liverpool, Liverpool, L69 3BX, UK

${ }^{3}$ Centre of Excellence in Long-acting Therapeutics (CELT), University of Liverpool, Liverpool, L7 3NY, UK

\section{Author for correspondence:}

Dr Paul Curley

Department of Pharmacology and Therapeutics

Centre of Excellence in Long-acting Therapeutics (CELT)

University of Liverpool

United Kingdom

Tel: +44 (0) 1517958560

E-mail: pcurley@liverpool.ac.uk

Key words: COVID-19, niclosamide, LC-MS/MS, plasma, SARS-CoV-2

\section{Conflicts of interest statement}

$A O$ and SR have received research funding from AstraZeneca and ViiV and consultancies from Gilead; $A O$ has additionally received funding from Merck and Janssen and consultancies from ViiV and Merck not related to the current paper. No other conflicts are declared by the authors.

\section{Funding}

This work was funded by UKRI using funding repositioned from EP/R024804/1 as part of the UK emergency response to COVID-19. The authors also acknowledge research funding from EPSRC (EP/S012265/1), NIH (R01Al134091; R24Al118397), European Commission (761104) and Unitaid (project LONGEVITY). 


\section{Abstract}

The SARS-CoV-2 pandemic has spread at an unprecedented rate, and repurposing opportunities have been intensively studied with only limited success to date. If successful, repurposing will allow interventions to become more rapidly available than development of new chemical entities. Niclosamide has been proposed as a candidate for repurposing for SARS-CoV-2 based upon the observation that it is amongst the most potent antiviral molecules evaluated in vitro. To investigate the pharmacokinetics of niclosamide, reliable, reproducible and sensitive bioanalytical assays are required. Here, a liquid chromatography tandem mass spectrometry assay is presented which was linear from $31.25-2000 \mathrm{ng} / \mathrm{mL}$ (high dynamic range) and 0.78-100 $\mathrm{ng} / \mathrm{mL}$ (low dynamic range). Accuracy and precision ranged between $97.2 \%$ and $112.5 \%, 100.4 \%$ and $110.0 \%$, respectively. The presented assay should have utility in preclinical evaluation of the exposure-response relationship and may be adapted for later evaluation of niclosamide in clinical trials. 


\section{Introduction}

Niclosamide (5-chloro-N-(2-chloro-4-nitrophenyl)-2-hydroxybenzamide) is an Food and Drug Administration (FDA) approved anthelmintic drug that is listed as an Essential Medicine by the WHO [1]. It has been in use since the 1960s, primarily for treating tapeworm infections [2]. Niclosamide has been proposed as a candidate for repurposing for SARS-CoV-2 based upon the observation that it is amongst the most potent molecules evaluated in vitro with VeroE6 cells [3]. At the time of writing, no studies have assessed the efficacy of niclosamide by any route of administration in an animal model. However, 10 studies are currently listed on clinicaltrials.gov describing trials of either oral or inhaled niclosamide formulations ( $\underline{w w w . c l i n i c a l t r i a l s . o r g})$.

The putative mechanism of action for niclosamide is not currently fully understood for SARS-CoV-2 but preliminary data have started to emerge within the preprint literature [4] A thorough understanding of the mechanism of action is critical to rationalize whether in vitro observations can be expected to lead to effects in vivo. Successful development of antiviral interventions for SARS-CoV2 will also require a robust assessment of the pharmacokinetic-pharmacodynamic relationship in preclinical models and patients, and many groups have called for better application of the principles of antiviral pharmacology during candidate selection for COVID-19 $[3,5,6]$. To better understand the exposure-response relationship, robust and validated bioanalytical methods are required to accurately determine the pharmacokinetics in preclinical and clinical studies.

Many existing methods for the analysis of niclosamide rely on HPLC systems [7-9]. Such methods are susceptible to interferences by other chemicals and are limited by poor sensitivity. While LC/MS MS methods for the detection of niclosamide have been developed; many are specific to matrices not representative for in vivo samples (e.g. water or cell culture media), or lack the dynamic range required for application to pharmacokinetic (PK) studies [10-12]. The potential application of niclosamide to SARS-CoV-2 therapy presents a bioanalytical challenge because of the paucity of data available publicly for the pharmacokinetics following administration to humans [13]. As such, development of 
a rapid, sensitive and versatile method to determine niclosamide concentrations in biological matrices is warranted.

The assay presented here was developed and validated in accordance with FDA guidelines [14]. Criteria such as linearity, accuracy, precision, selectivity (ensuring detection of the analyte and not an endogenous compound within the sample matrix) and recovery were assessed. The presented assay has application in pre-clinical evaluation as a SARS-CoV-2 antiviral intervention and may be adaptable to human matrices for future clinical evaluation if warranted. 


\section{Materials and Methods}

\section{Materials}

Niclosamide and the internal standard (IS) tizoxanide were purchased from Stratech Scientific Ltd (Cambridge, UK). Drug free Sprague Dawley plasma with lithium heparin was purchased from VWR International (PA, USA). LCMS grade acetonitrile (ACN) was purchased from Fisher Scientific (MA,USA). All other consumables were purchased from Merck Life Science UK LTD (Gillingham, UK) and were of LC-MS grade.

\section{Tuning and Calibration}

Detection of niclosamide was conducted using a QTRAP 6500+ Mass Spectrometer coupled with an Exion AD liquid chromatography system (SCIEX, UK). Tuning was performed using direct infusion (10 $\mu \mathrm{L} / \mathrm{min}$ ) of a $10 \mathrm{ng} / \mathrm{mL}$ stock of niclosamide with $\mathrm{H}_{2} \mathrm{O}$ :methanol (50:50). Ionisation was achieved via heated electron spray ionisation in the negative mode. The multiple reaction monitoring mode was selected for quantification of thea analytes, for which the precursors to production ion transitions were as follows: niclosamide $324.913 \rightarrow 170.8$ and $324.913 \rightarrow 288.8$. MS parameter voltage settings (DP, EP, CE, and CXP) were optimised to achieve the highest signal intensity.

\section{Chromatographic_separation}

Chromatographic separation was achieved using a multistep gradient (Table 1) with a Kinetex ${ }^{\circledR} \mathrm{C} 18$ column (100x2mm, $2.6 \mu \mathrm{M})$ obtained from Phenomenex (Macclesfield, UK). Mobile phases A and B were $\mathrm{H}_{2} \mathrm{O}+0.05 \%$ formic acid and $\mathrm{ACN}+0.05 \%$ formic acid, respectively. The assay was conducted over 3.5 minutes at a flow rate of $400 \mu \mathrm{l} / \mathrm{min}$, column temperature $25^{\circ} \mathrm{C}$. Data acquisition and chromatography analysis were carried out using the Analyst Software.

\section{Preparation of standards and controls}

Stock solutions of niclosamide and the IS tizoxanide were prepared at a concentration of $1 \mathrm{mg} / \mathrm{mL}$ in $\mathrm{ACN}$ and stored in glass vials at $4^{\circ} \mathrm{C}$. Working standards of niclosamide were prepared in rat plasma 
via serial dilution ranging from 31.25 to $2000 \mathrm{ng} / \mathrm{mL}$ for the high dynamic range or 0.78 to $100 \mathrm{ng} / \mathrm{mL}$ for the low dynamic range. Quality control samples (QC) of 75, 750 and $1500 \mathrm{ng} / \mathrm{mL}$ or 4,40 and 80 $\mathrm{ng} / \mathrm{mL}$ were prepared, respectively. The IS working solution $(10 \mathrm{ng} / \mathrm{mL})$ was prepared by diluting its stock solution with ACN.

\section{Extraction procedures}

Extraction was performed using a protein precipitation method. For the high dynamic range, a total volume of $100 \mu \mathrm{l}$ of sample, standard or QC was transferred to glass vials where $500 \mu \mathrm{L}$ of $100 \% \mathrm{ACN}$ containing $10 \mathrm{ng} / \mathrm{mL}$ of IS was added. Following mixing, samples were centrifuged at $3500 \mathrm{~g}$ for 5 minutes at room temperature. Thereafter, $50 \mu \mathrm{l}$ of the supernatant was directly transferred into 200 $\mu \mathrm{l}$ chromatography vials. A further $50 \mu \mathrm{l}$ of $\mathrm{H}_{2} \mathrm{O}$ was added and $1 \mu \mathrm{l}$ of each sample was injected into the LC-MS/MS system for analysis.

Similarly, for the low dynamic range a total volume of $100 \mu \mathrm{l}$ of sample, standard or QC was transferred to glass vials where $500 \mu \mathrm{L}$ of $100 \%$ ACN containing $10 \mathrm{ng} / \mathrm{mL}$ of IS was added. Following mixing, samples were centrifuged at $3500 \mathrm{~g}$ for 5 minutes at room temperature. $500 \mu \mathrm{L}$ of supernatant fraction was transferred to a fresh glass vial and evaporated to dryness under a gentle stream of nitrogen. The pelleted residue was reconstituted with $100 \mu \mathrm{L}$ solution of $\mathrm{H} 2 \mathrm{O}: \mathrm{ACN}$ (50:50) and vortexed. Subsequently, $50 \mu \mathrm{l}$ of the sample was then transferred into $200 \mu \mathrm{l}$ chromatography vials. Thereafter $1 \mu \mathrm{l}$ of each sample was injected into the LC-MS/MS system for analysis.

\section{Assay Validation}

The assay was validated according to the FDA guidelines for the development of bioanalytical assays [14] The following criteria were assessed: linearity, recovery, selectivity, accuracy, precision and interassay as well as intra-assay variability. 


\section{Linearity}

Linearity was assessed by three independent preparations of a calibration standard curve for each analyte. The calibration curve was obtained by plotting the ratio of peak area of analyte to the peak area of IS. Maximum allowed deviation of standards was set at $15 \%$ from the expected value, excluding the lower limit of quantification (LLOQ) where deviation was set at no more than $20 \%$.

\section{Recovery}

Recovery experiments were performed by comparing the results for extracted samples of each compound at three concentrations (same as high, medium and low QCs) with non-extracted standards that were taken to represent $100 \%$ recovery.

\section{Selectivity}

The degree of interference from the matrix (due to potential interfering substances including endogenous matrix components, metabolites and decomposition products) was assessed via comparison of extracted blank samples with the lowest point of the standard curve, the LLOQ. The LLOQ was a minimum of five-times greater than the background signal.

\section{Accuracy \& precision}

The intra-day and inter-day precision and accuracy of the method were determined with QC samples at three different concentrations $(\mathrm{N}=3)$ on the same day and on three different days. The calculated mean concentration relative to the nominal concentration was used to express accuracy (\% variability of accuracy $=$ error/stated value*100). The relative standard deviation was calculated from the QC values and used to estimate the precision (\%variation of precision $=$ standard deviation $/ \mathrm{mean}$ assay value*100). Acceptable variation for accuracy and precision was set at $15 \%$ and at $20 \%$ for the lower concentrations. 


\section{Results}

\section{Method development}

The optimised global settings were: Ion spray voltage $-4500 \mathrm{~V}$, Curtain gas 25 , source temperature $450^{\circ} \mathrm{C}$ and lon source gas (GS1/GS2) 50.00. MS parameter voltage settings for niclosamide were finetuned for maximum sensitivity, and parent ion transitions (MRM mode) were selected to afford the best response for the spectrum analysis as shown in the table below.

\section{Linearity}

The standard curves were linear in the concentration range between $31.25-2000 \mathrm{ng} / \mathrm{mL}$ and $0.78-100$ $\mathrm{ng} / \mathrm{mL}$ (Fig 1). The average correlations of calibration curve were found to be acceptable (Pearson's coefficient of determination $\mathrm{R}^{2}>0.99$ ). The peak area ratio (analyte to IS; variation of IS was $<15 \%$ in each run) was proportional to the stated concentration ranges.

\section{Extraction Recovery}

The recovery was evaluated using three replicates of QC samples at three concentration levels (the same concentrations as QC sample) in rat plasma. The results (Fig 2) showed that the recoveries at all three concentrations within both methods to be $>70 \%$. The mean recovery (across the 3 QCs) in the high dynamic range (Fig 2A) were 75\% (standard deviation 3.17) for the low QC, 75\% (standard deviation 2.95) for the mid QC, and 74\% (standard deviation 1.84) for the high QC. Recoveries for the low dynamic range (Fig 2B) were 97\% (standard deviation 2.05) for the low QC, 97\% (standard deviation 1.59) for the mid QC, and 97\% (standard deviation 1.33) for the high QC.

\section{Selectivity}

The matrix effect of plasma was examined by comparing extracted blank plasma to extracted plasma spiked with tizoxanide. For the high dynamic range figure $3 \mathrm{~A}$ shows the chromatogram produced by the extracted blank. There was no visible peak (area NA) at the retention time of niclosamide (2.5 minutes). FDA guidelines require the lower limit of quantification produce a peak area of at least five- 
fold greater than that observed in the blank matrix. Figure 3B shows the peak produced from the lower limit of quantification $(31.25 \mathrm{ng} / \mathrm{mL})$. The peak area is 2960 , which complies with FDA guidelines. Figure $3 C$ shows the peak (354170) produced by the highest standard $(2000 \mathrm{ng} / \mathrm{mL})$. The bottom panels of figure $3(D, E, F)$ for the low dynamic range also complied with FDA guidelines. Peak areas were as follows, 26518, 207766 and 25351511, for the extracted blank, the lower limit of quantification $(0.78 \mathrm{ng} / \mathrm{mL})$ and the highest standard $(100 \mathrm{ng} / \mathrm{mL})$, respectively. Additionally, the signal produced by the IS showed no interference with niclosamide in either methods.

\section{Accuracy \& precision}

The variability within and between assays was calculated to demonstrate that the accuracy and precision were maintained across repetitions of the assay. Table 3 shows the variance of accuracy and precision calculated from mean values of three repetitions of the assay (low QC, medium QC and high QC) either from the same experiment (intra-day) or an average of three separate experiments (interday). These data demonstrate that the precision and accuracy values were in the acceptance range $(<15 \%)$. The intra and inter-day precision was $\leq 3.42 \%$ and $\leq 10 \%$ for niclosamide. The intra and interday accuracy was $\leq 12.5 \%$ and $\leq 2.9 \%$ for niclosamide. 


\section{Discussion}

The SARS-CoV-2 pandemic has spread at an unprecedented rate, and repurposing opportunities have been intensively studied with only limited success to date. If successful, this approach will allow interventions to become rapidly available but a thorough understanding of the exposure-response relationship is required to improve the chances of success [3]. Robust bioanalytical methods underpin this understanding, providing a basis for translation of candidates to clinical evaluation. However, ultimate utility can only be confirmed in large and appropriately controlled randomized clinical trials, and drugs should not be used until such data are rigorously assessed with approval by appropriate agencies.

The assay presented here was developed for use in preclinical assessment of niclosamide pharmacokinetics in rats. Validation was conducted in plasma satisfying FDA bioanalytical method development guidelines, demonstrating good accuracy, precision and linearity. This assay represents an excellent starting point for researchers seeking to develop niclosamide assays. It should be noted that a very low signal was produced by blank samples, and the lower limit of detection was not fully evaluated in this study. Therefore, the true limit of the assay may be lower than the range reported here and this could be further optimized if required. The presented assay is currently being adapted to quantify niclosamide from plasma from other preclinical species and human plasma. Additional work is also underway to adapt the assay for quantification in other tissues such as lung and nasal turbinate. However, due to the more complex nature of tissue homogenates considerable additional optimization may be required. Tissue homogenate has a different protein composition compared to plasma and common proteins may be in different abundance. Niclosamide has been shown to bind to various endogenous proteins including hemoglobin, human serum albumin, and globulin demonstrating the importance of assessing any potential change in matrix when adapting this assay $[15,16]$ 
Examples of contemporary assays demonstrate niclosamide detection in a variety of matrices. The sensitivity typically falls in the range of approximately $1-40 \mathrm{ng} / \mathrm{mL}$ with an upper limit of $1000-8000$ $\mathrm{ng} / \mathrm{mL}[7,11,13]$. Although assays have been reported with lower sensitivity and higher detection limits, these assays are limited by the dynamic range. For example, Wang et al describe a method with a range of $0.02-12.5 \mathrm{ng} / \mathrm{mL}$ for in vitro applications [10]. While the lower limit of quantification is better than the assay presented here, the upper limit is not suitable for application to in vivo pharmacokinetic evaluation. Additionally, cell culture media is a considerably less complex matrix than plasma.

In summary, the optimization of a robust assay is described, providing a simple and sensitive LCMS/MS assay for application in preclinical assessments of niclosamide. The final assay conformed to FDA bioanalytical development guidelines and may be used to further the ongoing investigations into the utility of niclosamide against SARS-CoV-2, irrespective of formulation or route of administration. 


\section{References}

1. Organization, W.H., World Health Organization model list of essential medicines: 21st list 2019. 2019, World Health Organization.

2. Pawlowski, Z., J. Allan, and E. Sarti, Control of Taenia solium taeniasis/cysticercosis: from research towards implementation. Int J Parasitol, 2005. 35(11-12): p. 1221-32.

3. Arshad, U., et al., Prioritisation of potential anti-SARS-CoV-2 drug repurposing opportunities based on ability to achieve adequate target site concentrations derived from their established human pharmacokinetics. medRxiv, 2020.

4. Prabhakara, C., et al., Niclosamide inhibits SARS-CoV2 entry by blocking internalization through pH-dependent CLIC/GEEC endocytic pathway. bioRxiv, 2020.

5. Venisse, N., et al., Concerns about pharmacokinetic (PK) and pharmacokineticpharmacodynamic (PK-PD) studies in the new therapeutic area of COVID-19 infection. Antiviral Research, 2020: p. 104866.

6. Alexander, S., et al., A rational roadmap for SARS-CoV-2/COVID-19 pharmacotherapeutic research and development. IUPHAR review "XXX". British Journal of Pharmacology, 2020.

7. Lin, C.K., et al., Preclinical evaluation of a nanoformulated antihelminthic, niclosamide, in ovarian cancer. Oncotarget, 2016. 7(8): p. 8993-9006.

8. Cholifah, S., W. Farina Kartinasari, and G. Indrayanto, Simultaneous HPLC Determination of Levamisole Hydrochloride and Anhydrous Niclosamide in Veterinary Powders, and its Validation. Journal of Liquid Chromatography \& Related Technologies, 2007. 31(2): p. 281291.

9. Chang, Y.-W., et al., Pharmacokinetics of anti-SARS-CoV agent niclosamide and its analogs in rats. Journal of Food and Drug Analysis, 2006. 14(4).

10. Wang, G., et al., Fast and scalable lipid nanoparticle formulation of niclosamide (nano NCM) effectively inhibits SARS-CoV-2 replication in vitro. bioRxiv, 2020.

11. Doran, G. and M.M. Stevens, Simultaneous determination of niclosamide and its degradates in water by LC-MS/MS. Analytical Methods, 2014. 6(17): p. 6871-6877.

12. Jiang, H., et al., Simultaneous determination of pentachlorophenol, niclosamide and fenpropathrin in fishpond water using an LC-MS/MS method for forensic investigation. Analytical Methods, 2013. 5(1): p. 111-115.

13. Schweizer, M.T., et al., A phase I study of niclosamide in combination with enzalutamide in men with castration-resistant prostate cancer. PLoS One, 2018. 13(6): p. e0198389.

14. (FDA), U.S.F.D.A., Bioanalytical Method Validation Guidance for Industry, C.f.D.E.a. Research and C.f.V. Medicine, Editors. 2020.

15. Maltas, E., Binding interactions of niclosamide with serum proteins. J Food Drug Anal, 2014. 22(4): p. 549-555.

16. Zhou, W., S. Yang, and P.G. Wang, Matrix effects and application of matrix effect factor. Bioanalysis, 2017. 9(23): p. 1839-1844. 


\section{Tables}

Table 1 The operating chromatographic conditions

\begin{tabular}{ccc}
\hline Time (minutes) & $\begin{array}{c}\text { Mobile Phase A (\%) } \\
\mathrm{H}_{2} \mathrm{O} 0.05 \% \text { formic acid }\end{array}$ & $\begin{array}{c}\text { Mobile Phase B (\%) } \\
\text { ACN 0.05\% formic acid }\end{array}$ \\
\hline $\mathbf{0 . 0}$ & 95 & 5 \\
\hline $\mathbf{0 . 5}$ & 95 & 5 \\
\hline $\mathbf{1}$ & & 80 \\
\hline $\mathbf{2 . 5}$ & 20 & \\
\hline $\mathbf{3 . 0}$ & 5 & 95 \\
\hline $\mathbf{3 . 0 1}$ & 5 & \\
\hline $\mathbf{3 . 5}$ & 95 & 95 \\
\hline
\end{tabular}

Table 2 shows the final source parameters for used for the detection of niclosamide and tizoxanide (IS).

\begin{tabular}{|c|c|c|c|c|c|c|}
\hline Parent $(\mathrm{m} / \mathrm{z})$ & $\begin{array}{l}\text { Product } \\
(\mathrm{m} / \mathrm{z})\end{array}$ & $\begin{array}{c}\text { RT } \\
\text { (Min) }\end{array}$ & $\begin{array}{c}\text { DP } \\
\text { (Volts) }\end{array}$ & $\begin{array}{c}\text { EP } \\
\text { (Volts) }\end{array}$ & $\begin{array}{c}\text { CE } \\
\text { (Volts) }\end{array}$ & $\begin{array}{c}\text { CXP } \\
\text { (Volts) }\end{array}$ \\
\hline Niclosamide & 113.900 & 1.97 & -34 & -10 & -28 & -11 \\
\hline 263.965 & 216.800 & 1.97 & -34 & -10 & -18 & -23 \\
\hline Tizoxanide (IS) & 170.8 & 2.20 & -140 & -10 & -32 & -23 \\
\hline 324.913 & 288.8 & 2.20 & -140 & -10 & -24 & -27 \\
\hline
\end{tabular}


Table 3 Intra-day and Inter-day accuracy and precision for niclosamide in rat plasma $(\mathrm{n}=3)$

\begin{tabular}{|c|c|c|c|c|c|c|c|}
\hline & & \multicolumn{4}{|c|}{ Intra-day } & \multicolumn{2}{|c|}{ Inter-day } \\
\hline & & \multirow{3}{*}{$\begin{array}{c}\text { Average } \pm \text { SD } \\
\text { (ng/mL) }\end{array}$} & & & \multirow{3}{*}{$\begin{array}{c}\text { Average } \pm \text { SD } \\
(\mathrm{ng} / \mathrm{mL})\end{array}$} & & \\
\hline & & & Variance of & Variance of & & Variance of & Variance of \\
\hline & & & accuracy (\%) & precision (\%) & & accuracy (\%) & precision (\%) \\
\hline \multirow{4}{*}{$\begin{array}{l}\text { Niclosamide High } \\
\text { Dynamic Range }\end{array}$} & High (1500 ng/mL) & $1518.0 \pm 28.2$ & 1.2 & 1.86 & $1514.2 \pm 3.33$ & 0.94 & 0.22 \\
\hline & Medium (750ng/mL) & $741.5 \pm 14.8$ & -1.12 & 1.9 & $751.2 \pm 8.4$ & 0.2 & 1.12 \\
\hline & Low (75ng/mL) & $72.8 \pm 2.0$ & -2.8 & 2.74 & $75.1 \pm 1.9$ & 0.1 & 2.5 \\
\hline & High (80 ng/mL) & $82.2 \pm 2.8$ & 2.75 & 3.42 & $78.3 \pm 5.6$ & -2.1 & 7.1 \\
\hline \multirow{2}{*}{$\begin{array}{l}\text { Niclosamide Low } \\
\text { Dynamic Range }\end{array}$} & Medium (40 ng/mL) & $44.9 \pm 0.1$ & 12.3 & 0.4 & $41.1 \pm 3.9$ & 2.9 & 9.6 \\
\hline & Low (4ng/mL) & $4.5 \pm 0.1$ & 12.5 & 2.7 & $4.1 \pm 0.4$ & 1.9 & 10.0 \\
\hline
\end{tabular}




\section{Figure legends}

Figure 1 Linearity of the bioanalytical method. A) shows an example of the linearity using the high concentration range $(31.25-2000 \mathrm{ng} / \mathrm{mL})$. B) shows an example of the linearity using the low concentration range $(0.78-100 \mathrm{ng} / \mathrm{mL}) . \mathrm{R}^{2}$ values for the line of best fit are also shown.

Figure 2 Niclosamide recovery. A) shows the percentage recovery for high dynamic range and B) shows the percentage recovery for low dynamic range. Data is show percentage of unextracted standards \pm percentage standard deviation.

Figure 3 shows representative chromatograms from the high dynamic range method for the blank plasma (A), lower limit of quantification $(31.25 \mathrm{ng} / \mathrm{mL})(B)$ and the highest standard $(2000 \mathrm{ng} / \mathrm{mL})(C)$. Representative chromatograms for the low dynamic range method are shown for the blank plasma (D), lower limit of quantification $(0.78 \mathrm{ng} / \mathrm{mL})(E)$ and the highest standard $(100 \mathrm{ng} / \mathrm{mL})(\mathrm{F})$. The peak produced by niclosamide had a retention time of 2.5 minutes and the peak produced by the IS tizoxanide had a retention time 2.09 minutes. 


\section{Figures}

\section{Figure 1}

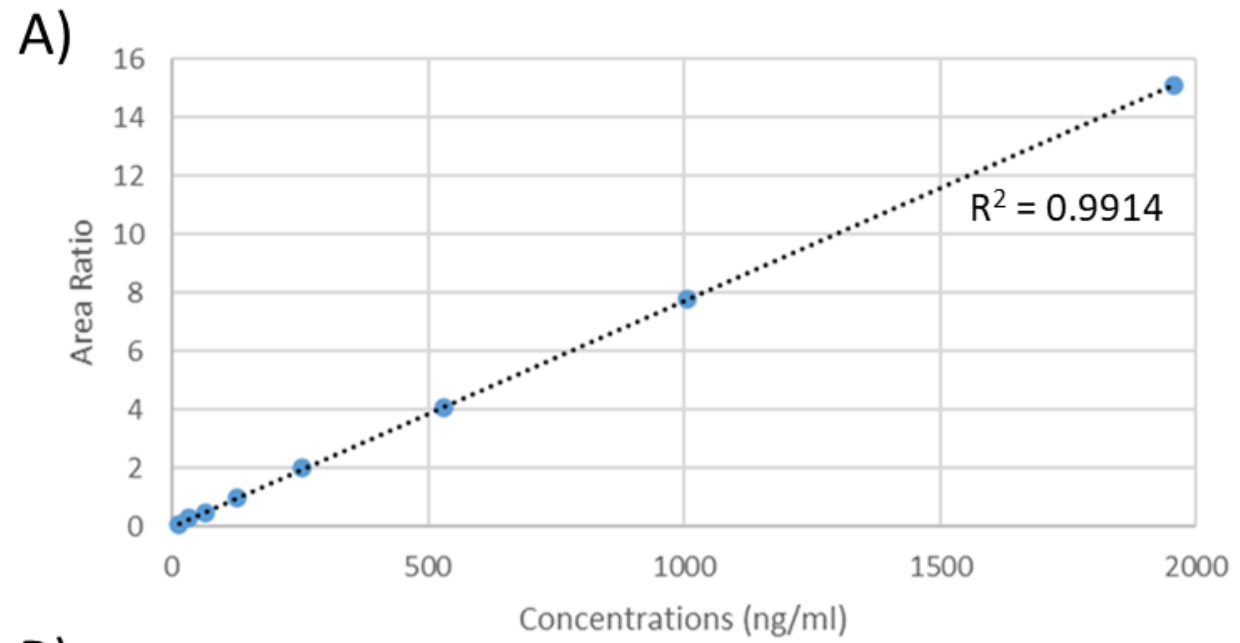

B)

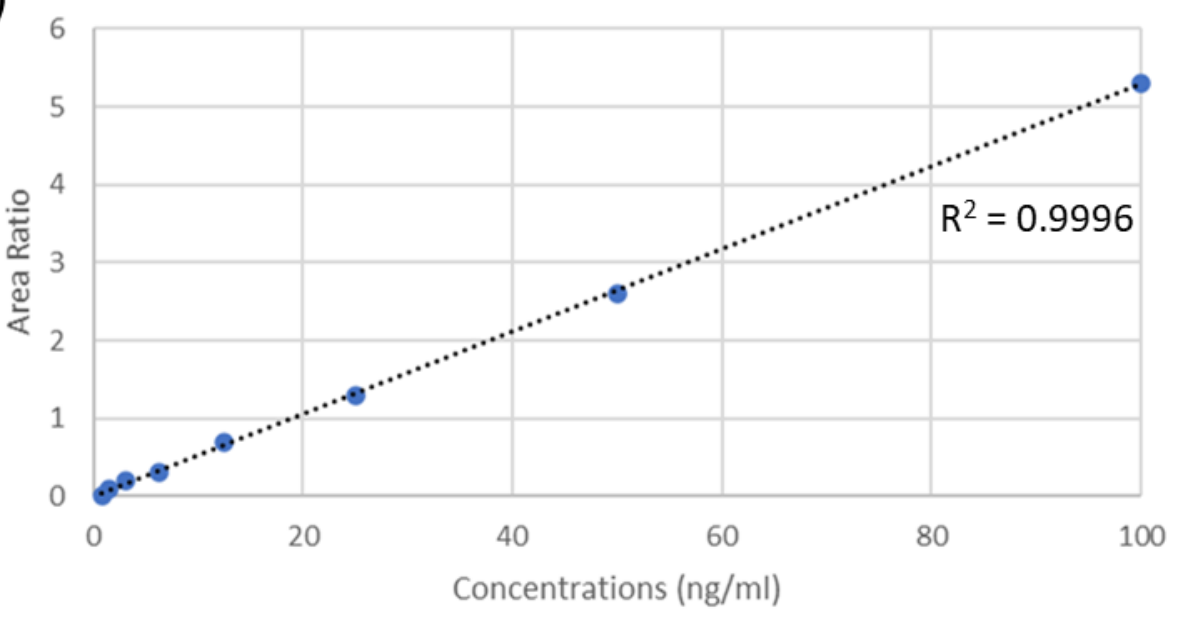




\section{Figure 2}

A) 100

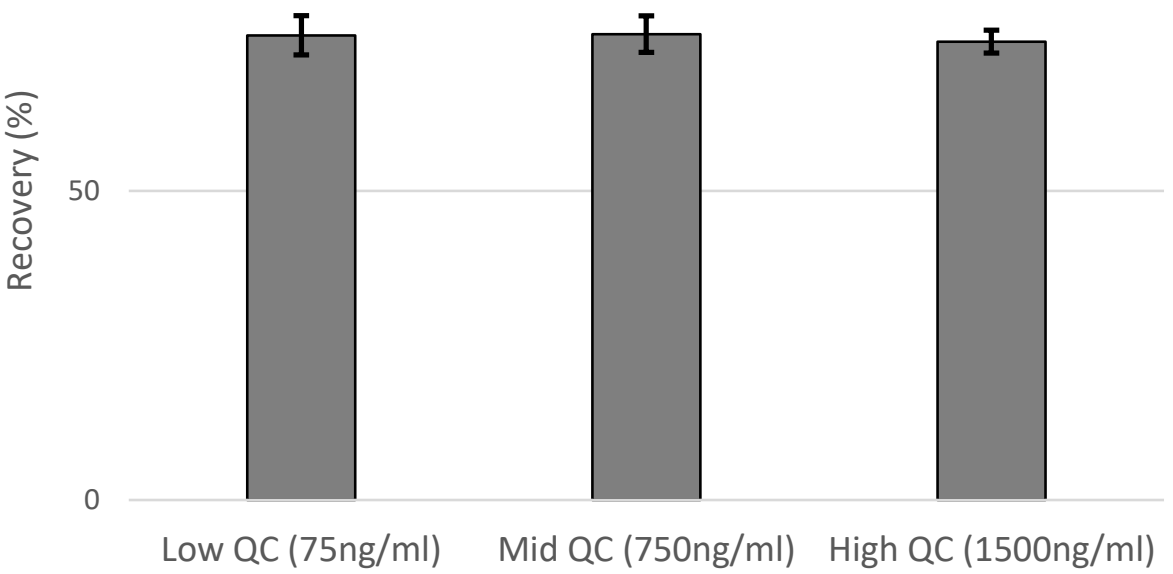

B)

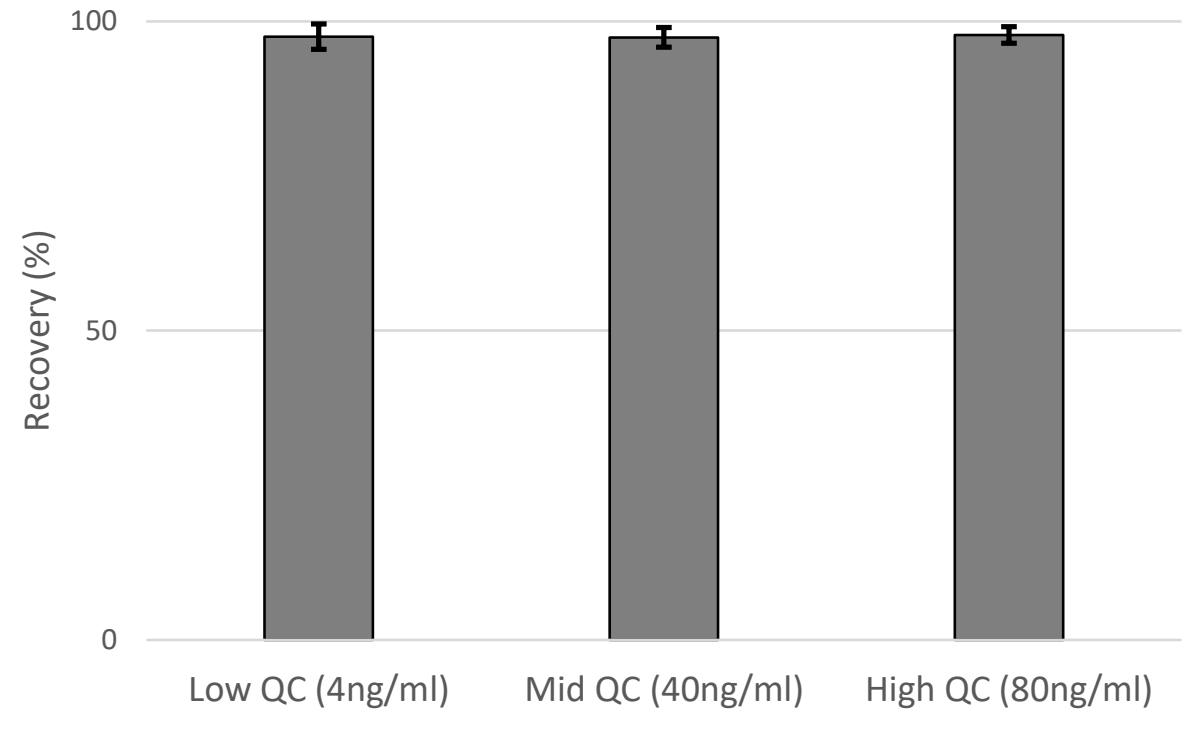


Figure 3
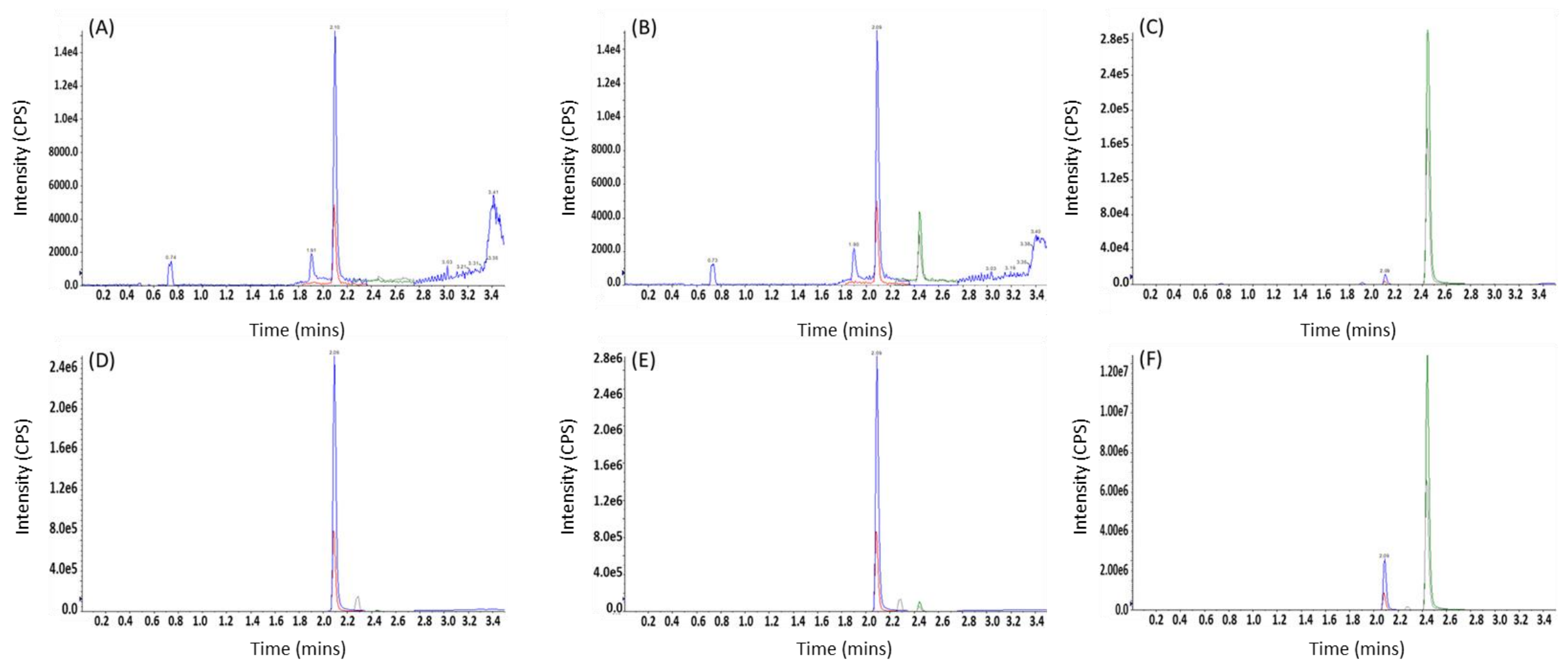